\title{
PATHOPHYSIOLOGICAL ASPECTS AS THE OBJECT OF TREATMENT TACTICS IN ACUTE PACREATITIS (REVIEW)
}

\author{
Kryvoruchko Igor, Drozdova Anastasiya, Goncharova Natalya \\ Kharkiv National Medical University \\ https://doi.org/10.35339/ic.8.2.87-94
}

\begin{abstract}
Summary
The review presents a modern view on the features of the course and treatment of acute pancreatitis, based on a cascade of pathophysiological mechanisms of this disease. A number of concepts of acute pancreatitis development and course are based on randomized prospective and retrospective studies of this problem are reviewed. Attention is paid to the mechanisms of organ failure development in acute pancreatitis. In accordance with the above, the main positions of treatment measures for acute pancreatitis, which are based on the principles of tactics "step-up approach" were featured. Among them, attention is paid to the features of the conservative treatment program, minimally invasive surgical interventions, as well as the management of the postoperative period of patients. Minimally invasive surgical interventions perform the main tasks of surgical treatment in acute pancreatitis, but significantly reduce surgical trauma compared to "open" methods. Adequate management of the postoperative period of patients is carried out through the implementation of protocols "fast-track surgery". Keywords: acute pancreatitis, organ failure, tactics "step-up approach".
\end{abstract}

Acute pancreatitis is a sudden inflammatory process in the pancreas with possible involvement of the adjacent organs or even organs of other systems $[1,2]$. Today, the incidence of acute pancreatitis is ahead of all urgent diseases of the abdominal cavity.

Acute pancreatitis causes major morbidity and mortality. According to global estimates, the incidence of acute pancreatitis was shown to be 33-74 cases per 100000 person a years and a mortality of $1-60$ per 100000 persons a years. In European countries acute pancreatitis occurs from 4.6 to 100 cases per 100 thousand population [3-5]. According to British pancreatologists, the incidence of acute pancreatitis ranges from 15 to 42 cases per 100 thousand population during a year, with an annual increase of $2.7 \%$ [1].

Among the leading causes of acute pancreatitis the authors highlight the presence of gallstones and alcohol abuse. Moreover, in women over 60 years of age the leading role is played by biliary pancreatitis, and in men alcoholic predo-

\footnotetext{
Corresponding Author:

Drozdova Anastasiya, MD, PhD student

of the Department of Surgery \#2, Kharkiv National

Medical University, Kharkiv, Ukraine.

E-mail: anasteisha.06@gmail.com
}

minates. In about $30 \%$ of cases it is an idiopathic acute pancreatitis (when it is not possible to identify the leading etiological factor in the development of the disease) $[1,2,4,5]$.

Pancreas is essentially the first target organ of autoenzyme aggression and the pathophysiology of acute pancreatitis, despite the etiological factor, consists in activation and release of pancreatic enzymes into the interstitial space, autodigestion and multiple dysfunction of organs (MODS) of active systems after release mediators. In the early stage of enzyme release and as a manifestation of distant autoenzyme aggression, a picture of local (in the parenchyma of the gland) and widespread (in adjacent organs) vasculitis develops. Inflammatory mediators in this case acquire destructive functions, organ failure develops, including intestinal endoxemia and bacterial translocation, which is the main mechanism of septic complications in acute pancreatitis. Regarding the role of the intestine in the pathogenesis of the disease, this is confirmed by the fact that most of the bacteria that cause secondary pancreatic infection are of enteral origin.

Acute pancreatitis is a dynamic process with a variety of pathophysiological mechanisms of local and systemic complications. Adequate 
assessment of the state of the body's defense and awareness of the interaction between the various components of the immune system allows quick identification of emerging disorders and determine the strategy of targeted therapy [1-3].

The tactics, methods and ways of treating acute pancreatitis and its complications have been discussed in the literature for a long time, which leads to the formation of opposing views on this issue. In such a situation, it is important and relevant to timely assess the severity of the patient's condition, so that it is possible to predict the further development of the disease and accordingly choose the right treatment tactics. According to the international recommendations, severity of the patient's condition should be determined within the first 24 hours after hospitalization of the patient to the hospital. Therefore, the use of world-famous scales and prognostic systems, in particular Marshall's multiorgan dysfunction scale, SOFA scale, APACHE II scale, and BISAP scale, plays an important role in adequately assessing the severity of acute pancreatitis in a patient.

Acute pancreatitis is characterized by a wide range of clinical manifestations from mild to severe necrotic inflammation, which occurs with the systemic inflammatory response syndrome (SIRS), MODS and then organ failure. Severe acute pancreatitis is characterized by the presence of persistent MODS, as noted above, which largely determines the outcome and the possibility of death in the patient. Organ failure, as a generic term, can be defined as significant functional impairment of an organ system that is critical to sustenance of life. Severity of organ dysfunction can be quantified based on the parameter best defining the primary function of that particular organ (e.g. partial pressure of arterial oxygen $(\mathrm{PaO} 2)$ for pulmonary function or serum creatinine for renal function). In the case of acute pancreatitis, 3 organ systems are considered most important (respiratory, renal and cardiovascular) which are most commonly involved. According to world studies, the frequency of organ failure in acute pancreatitis can be $8-20 \%[4,8,9-11]$. Risk factors for the development of organ failure in acute pancreatitis are age, the presence of comorbidities, obesity, triglyceride levels, etiology, the degree of local damage to the pancreas and genetic predisposition [12-14].

Although the development of organ failure and its consequences have long been confirmed in acute pancreatitis, there are separate concepts of primary organ failure, which develops early due to acute pancreatitis (aseptic inflammation) and may precede necrosis, and secondary organ failure, which develops due to infected pancreatic necrosis induced sepsis. Infection of necrotic tissue of the pancreas is an ominous harbinger of secondary organ failure development, which can cause "late" death in acute pancreatitis. It should be noted that the prognostic development of primary organ failure is much worse than secondary. Because primary organ failure develops so rapidly and leads to "early" death that the doctor has almost no time for treatment. In secondary organ failure, as a rule, the patient's condition allows for a number of therapeutic measures, after which the fight against sepsis comes to the fore $[4,10]$.

The main type of treatment of acute pancreatitis is complex conservative treatment and surgery in the presence of indications for it.

The main goal of treatment tactics in acute pancreatitis is to reduce the likelihood of developing infected pancreatic necrosis and the possibility of death. Over the recent 10 years, the results of treatment of patients with acute pancreatitis have improved slightly, but the incidence of various infectious complications still remains high (ranging from 40 to $70 \%$ of cases), which in turn can lead to development of sepsis with the development of organ failure. The total mortality in severe forms of acute pancreatitis can reach $15 \%$ in sterile forms and $30 \%$ in infected. Secondary pancreatic infection is a further factor that often leads to adverse effects, and is diagnosed in approximately $40 \%$ of patients and is associated with high mortality, which exceeds $40 \%$ with the development of systemic complications.

At present, the world-wide accepted indication for surgical intervention in acute pancreatitis is development of a secondary pancreatic infection. According to the authors, indications for surgical treatment of acute pancreatitis are usually deterioration of the general condition of the patient on the background of conservative therapy (persistent hyperthermia, increased pain, the appearance of positive peritoneal symptoms), the development of purulent-septic complications, peritonitis $[2,3]$.

It is known that the principles of surgical interventions in pancreatic necrosis were laid down by B. Moynihan in 1925, and the main surgical methods for the control of secondary pancreatic infection and sepsis over the past 40 years included: 1) "open method" of treatment in the form of necrosectomy, sanitation and open management 
of the focus of infection; 2) necrosectomy with routine relaparotomies and re-sanitation of the source of infection; 3) "closed technique" with necrosectomy, drainage and with or without continuous washing $[2,6]$. But even today the principles of treatment of necrotic pancreatitis and the role of surgery remain controversial. In the 1990s, more than $60 \%$ of patients with acute pancreatitis were treated with open surgical interventions. In 1991, E.L. Bradley and K. Allen recommended conservative treatment of sterile necrosis of the pancreas in selected cases, and M. Gagner was the first to perform and describe minimally invasive videolaparoscopic surgical treatment of secondary pancreatic infection in 1996, including laparoscopic retrocolic, retroperitoneoscopic interventions $[2,5,6]$.

Subsequently, it was hypothesized that percutaneous drainage of infected pancreatic necrosis and fluid collectors may have a positive therapeutic effect. This recommendation was based on clinical observations, which indicated that there was no need for maximum removal of all necrotic tissue for successful treatment of patients with infected pancreatic necrosis. By draining infected fluid collectors, the authors proved that the clinical condition of patients can improve after these interventions, and necrotic tissues can be successfully treated in the subsequent immune system of the patient. That is, the purpose of drainage is to remove the infected fluid, not necrosis [5, 22].

Since 2010, the world community of pancreatologists has abandoned open surgical necrosectomy and initiated introduction of a "step-up approach" tactics to the surgical clinic. Because open necrosectomy led to the appearance of massive SIRS with the further development of organ failure and local purulent-septic complications. The use of minimally invasive techniques in the complex treatment of acute pancreatitis, such as percutaneous drainage, transluminal endoscopic necrosectomy through the stomach or duodenum, laparoscopic necrosectomy and retroperitoneal surgical drainage is becoming more common. Sometimes it is a primary drainage followed by necrosectomy, if necessary, or, in the case of acute biliary pancreatitis, during the first surgical intervention, the abdominal cavity and the omental bag are adequately sanitized, and during the second, a cholecystectomy is performed. This reflects the principles of "step-up approach" tactics in modern pancreatology. In some clinics, due to this tactic in the treatment of necrotic acute pancreatitis it is possible to reduce mortality in its infected forms to $15.4 \%[22,23]$.
An important component of the "step-up approach" tactics is modern conservative treatment, which allows significant limitation of the indications for the implementation of early surgical intervention in acute pancreatitis, and surgical methods should be used only for absolute indications. The main principles of conservative treatment of acute pancreatitis: correction of disorders of central hemodynamics and peripheral blood circulation, respiratory support, analgesia, decompression of the gastrointestinal tract, adequate protein and energy supply, prevention and treatment of purulent infection, suppression of secretory activity of the pancreas, hepatoprotection, prevention and treatment of intraabdominal hypertension syndrome. Non-narcotic analgesics are usually preferred to provide adequate analgesia. At the expressed pain syndrome, especially in combination with intestinal paresis, the extended epidural analgesia on ThVII-ThVIII levels by introduction of solutions of local anesthetics is widely used. Hemodynamic support during intensive care in conditions of toxemic and enzymatic shock begins with the correction of hypovolemia and dehydration, as well as microcirculation disorders. Central venous pressure is maintained at $100 \mathrm{~mm}$ of water column, diuresis - 40-60 ml/hour. At unstable hemodynamics and disturbance of perfusion of tissue at adequate liquid resuscitation use of vasopressors is shown. Respiratory support (oxygen therapy and artificial lung ventilation) in the conditions of intensive care unit is justified in respiratory failure $(\mathrm{SaO} 2<95 \%, \mathrm{PaO} 2<70 \mathrm{~mm} \mathrm{Hg})$. In order to block pancreatic secretion, somatostatin and polyvalent serine protease inhibitor unilastatin are used, which also reduces the synthesis of proinflammatory cytokines by immunocompetent cells and endothelium, reduces the activity of leukocyte elastases and lysosomal hydrolases, and other. Prevention and antibacterial therapy of infectious complications is carried out in accordance with the international recommendations of the IAP/APA for the treatment of acute pancreatitis. Antibiotics with a broad spectrum of action are prescribed, which are active against most probable pathogens of pancreatic infection and are able to penetrate into the tissue of the pancreas, gland secretion, parapancreatic tissue (mostly carbapenems) $[1,5,6,23,24]$.

The main task of surgical intervention is the timely evacuation of exudate from the abdominal cavity during enzymatic toxemia or necrotic areas of the gland, when they already exist, with minimal risk to the patient's life. 
Puncture-drainage methods are the next stage of the diagnostic and treatment algorithm in patients with acute necrotic pancreatitis. In some clinical cases, they can be considered as a direct alternative to surgery and can be successfully performed in $54 \%$ of cases under ultrasonographic or computed tomography control [25-27].

A number of authors position percutaneous drainage interventions as a temporary preoperative measure or a direct alternative to surgery in a limited group of patients. Installation of percutaneous drainage improves the demarcation of foci of pancreatic necrosis and reduces surgical trauma in the future. Drainage sizes 14-32 French are used for drainage. The catheter should be optimally placed in the left or right retroperitoneal space, depending on the location of the fluid collector. Some sources indicate that catheters of large diameters (up to 28Fr) may be more effective, given the morphological specifics of pancreatic and peripancreatic necrosis, which contain a large number of dense necrotic elements. The procedure of gradually increasing the size of drainage catheters, which allows you to evacuate necrotic tissue, is called percutaneous necro-sectomy $[2,5,6,23,25,27]$.

The transluminal endoscopic techniques occupy an important niche in the treatment of acute necrotic pancreatitis. The endoscopic method of treatment of peripancreatic fluid accumulations began its history in 1975, when the case of transgastric cyst drainage was first described in the literature. The results of research conducted in the early 2000s showed the first successes of the new technique: the success of drainage in $70-100 \%$. Of course, in recent decades, the endoscopic method has undergone a major evolution - from simple transgastric aspiration to transluminal necrosectomy using ultrasound monitoring and special stent systems. According to a number of authors, transgastric necrosectomy avoids dangerous complications associated with transcutaneous puncture (bleeding, perforation of hollow organs, formation of external pancreatic fistula), and provides a more direct approach to the foci of necrosis. The final stage of the intervention is the placement of stents (double-pigtail) 5-10 Fr, usually two, or a nasogastric catheter inserted into the cavity of the omental bag. Sanitation of the cavity should be carried out within 24 hours by irrigation with saline solution with a volume of 1 liter. Evaluation of endoscopic drainage results should be performed within the next 72 hours. Repeated endoscopic procedure should be performed in cases of no clinical improvement $[2,3,5,6,28]$.
As for videolaparoscopy, it performs both diagnostic and therapeutic tasks. When perfor-ming videolaparoscopy, the diagnosis of acute pancreatitis should be confirmed and other acute abdominal diseases should be ruled out $[23,25$, 28]. Significant signs of acute pancreatitis include edema of the mesenteric root of the colon, effusion with high amylase activity (2-3 times the activity of blood amylase), "vitreous edema" (serous infiltration of fatty tissue), the presence of foci of steatonecrosis (arising from the action of lipases and phospholipases). In favor of severe acute pancreatitis indicate hemorrhagic nature of the exudate (cherry, pink, brown), common areas of steatonecrosis, significant hemorrhagic infiltration of retroperitoneal tissue beyond the pancreas. The condition of the biliary system, in particular the gallbladder (whether it is tense, able to empty, or contains concretions) should also be assessed during the intervention. If the gallbladder is tense, unable to empty, the surgery must end with the unloading of the biliary system in the form of cholecystostomy.

The analysis of the literature shows that transabdominal necrosectomy techniques in terms of frequency of use in clinical practice are significantly inferior to retroperitoneoscopic. Laparoscopic access to the pancreas is similar to that of open surgery: through the gastrocolic ligament or mesocolon. The ability to achieve the most complete removal of necrotic tissue makes the method particularly attractive $[1,2,5,6,22,23,28,29]$.

A number of publications report the success of single-port laparoscopic necrosectomy and laparoscopically-assisted necrosectomy (handassisted) [29-36].

Video-Assisted Retroperitoneal Debridement (VARD) is the most common minimally invasive technique in the United States and the Netherlands. The undoubted advantage of the method is the lack of contact with the abdominal cavity, which prevents its infection. The technique was first described by Horvath et al. in 2001. VARD is an integral part of the step-up approach in the treatment of acute necrotic pancreatitis. Transcutaneous drainage is considered a mandatory step prior to VARD. Drainage of the left retroperitoneal space is technically possible in $95 \%$ of cases. The essence of the technique is subcostal access to the retroperitoneal space, the reference point for which is the retroperitoneal drainage. At achievement of necrotic masses the indirect necrosectomy is carried out. In the future, using a laparoscope by insufflation of carbon dioxide, a direct (video-assisted) part of the operation is performed [23, 33, 37-41]. 
The problem of comprehensive treatment of patients with acute pancreatitis remains relevant, as well as the problem of adequate management of this category of patients in the postoperative period. It is not news for surgeons and resuscitators that only half of the success of treatment of a patient with acute pancreatitis depends on timely intervention, and half still depends on adequate treatment in the postoperative period, because this pathology, as mentioned above, has a rather complex cascading pathophysiological mechanisms of development [42-48].

The application of the step-up approach in clinical practice is closely intertwined with the implementation of the concept of multimodal rehabilitation of surgical patients through the implementation of the protocols Enhanced Recovery After Surgery (ERAS) or "fast-track surgery". This helps to reduce the cost of treatment without compromising its quality. The ERAS concept envisages a set of measures in the periand postoperative period aimed at reducing the time of hospitalization and rehabilitation after the intervention. Since patients with acute pancreatitis are the category of patients who most often need long-term (what to hide, and sometimes prolonged) and costly inpatient treatment, attempts to implement the concept of ERAS during their treatment are relevant and cost-effective. The concept of "fast-track surgery" requires coordinated action of all medical staff, as well as full understanding between the patient and the doctor, who motivates and sets the patient's daily tasks. Studies of the pathophysiological mechanisms of any surgical intervention have shown that the key is the failure of physiological activity of internal organs in response to surgical stress. These changes in the functions of internal organs are mediated by metabolic changes caused by surgical trauma and the activation of a number of cascade systems. Multimodal rehabilitation with an emphasis on preoperative patient information, reduced response to surgery, optimized pain relief, early mobilization and early feeding reduced the number of complications, length of stay in the ward, and therefore the cost of treatment [48-50].

Declarations:

\section{Statement of Ethics}

The authors have no ethical conflicts to disclosure.

\section{Consent for publication}

All authors give their consent to publication.

\section{Disclosure Statement}

The authors have no potential conflicts of interest to disclosure.

\section{Funding Sources}

This study was not supported by any grants from public, commercial or other sectors.

\section{Data Transparency}

The data can be requested from the authors.

\section{References:}

1. Goodchild G., et al. (2009). Review Practical guide to the management of acute pancreatitis. Frontline Gastroenterology. 10. P. 292-299 DOI: 10.1136/flgastro-2018-101102

2. Gurusamy K.S., Belgaumkar A.P. et al. (2016). Interventions for necrotising pancreatitis (review). Cochrane Database of Systematic Reviews. Issue 4. Art. No.: CD011383. 83 p. DOI: 10.1002/ 14651858.CD011383.pub2.

3. Xiao AY, Tan ML, Wu LM, et al. (2016). Global incidence and mortality of pancreatic diseases: a systematic review, meta-analysis, and meta-regression of population-based cohort studies. Lancet Gastroenterol Hepatology. 1(1). P. 45-55. [PubMed: 28404111]

4. Pramod K. Garg, Vijay P. Singh. (2019). Organ Failure due to Systemic Injury in Acute Pancreatitis. Gastroenterology. Author manuscript. 156(7). P. 2008-2023. doi:10.1053/j.gastro.2018.12.041.

5. Leppaniemi Ari et al. (2019). 2019 WSES guideliners for the management of severe acute pancreatitis. World Journal of Emergency Surgery. 14:27. 20 p. https://doi.org/10.1186/s13017-019$0247-0$

6. Working Group IAP/APA Acute Pancreatitis Guidelines. IAP/APA evidence-based guidelines for the management of acute pancreatitis. (2013). Pancreatology. 13. 15 p. http://dx.doi.org/10.1016/ j.pan.2013.07.063

7. Peter A. Banks, Thomas L. Bollen et al. (2013). Classification of acute pancreatitis - 2012: revision of the Atlanta classification and definitions by international consensus. Pancreas. 62: P.102-111. DOI 10.1136/gutjnl-2012-302779

8. Hamada S, Masamune A, Kikuta K, et al. (2014). Nationwide epidemiological survey of acute pancreatitis in Japan. Pancreas. 43:1244-8. [PubMed: 25084001]

9. Kamal A, Sinha A, Hutfless SM, et al. (2017). Hospital admission volume does not impact the inhospital mortality of acute pancreatitis. HPB (Oxford). 19:21-28. [PubMed: 27887788] 
10. Padhan RK, Jain S, Agarwal S, et al. (2018). Primary and Secondary Organ Failures Cause Mortality Differentially in Acute Pancreatitis and Should be Distinguished. Pancreas. 47:302-307. [PubMed: 29401171].

11. Schepers NJ, Bakker OJ, Besselink MG, et al. (2018). Impact of characteristics of organ failure and infected necrosis on mortality in necrotising pancreatitis. 68(6): 1044-1051. doi: 10.1136/gutjnl2017-314657.

12. de-Madaria E, Martinez J, Sempere L, et al. (2008). Cytokine genotypes in acute pancreatitis: association with etiology, severity, and cytokine levels in blood. Pancreas. 37:295-301. [PubMed: 18815552]

13. Bishehsari F, Sharma A, Stello K, et al. (2012). TNF-alpha gene (TNFA) variants increase risk for multi- organ dysfunction syndrome (MODS) in acute pancreatitis. Pancreatology. 12:113-8. [PubMed: 22487520].

14. Yang Z, Qi X, Wu Q, et al. (2012). Lack of association between TNF-alpha gene promoter polymorphisms and pancreatitis: a meta-analysis. 503:229-34. [PubMed: 22579868]

15. Reintam A, Parm P, Kitus R, Starkopf J, Kern H. (2008). Gastrointestinal failure score in critically ill patients: a prospective observational study. Crit Care 2008; 12(4):R90. doi: 10.1186/cc6958

16. Blaser A, Malbrain M, Starkopf J, Fruhwald Jakob S, De Waele J, Braun J, et al. (2012). Gastrointestinal function in intensive care patients: Terminology, definitions and management Recommendations of the ESICM Working Group on Abdominal Problems. Intensive Care Med. 38:38494. doi: $10.1007 / \mathrm{s} 00134-011-2459-\mathrm{y}$

17. Hiroyuki Funaoka 1, Tatsuo Kanda, Hiroshi Fujii (2010). Intestinal fatty acid-binding protein (I-FABP) as a new biomarker for intestinal diseases. Review. 2010 Feb;58(2):162-8. PMID: 20229815

18. Li H, Chen Y, Huo F, Wang Y, Zhang D. (2017). Association between acute gastrointestinal injury and biomarkers of intestinal barrier function in critically ill patients. BMC Gastroenterology. 17:45 doi: 10.1186/s12876-017-0603-z

19. Piton G, Belon F, Cypriani B, Regnard J,Puyraveau M, Manzon C, et al. (2013). Enterocyte damage in critically ill patients isassociated with shock condition and 28-day mortality. Crit Care Med. 2013 Sep; 41(9):2169-76. doi: 10.1097/CCM.0b013e31828c26b5

20. Uzun O, Turkmen S, Eryigit U et al. (2014). Can Intestinal Fatty Acid Binding Protein (I-FABP) Be A Marker in the Diagnosis of Abdominal Pathology? Turkish J Emerg Med. 14(3): 99-103. https:// doi.org/10.5505/1304.7361.2014.15679; PMid:27355087 PMCid:PMC4909954

21. Voth, M., Lustenberger, T., Relja, B. et al. (2019). Is I-FABP not only a marker for the detection abdominal injury but also of hemorrhagic shock in severely injured trauma patients?. World J Emerg Surg 14, 49. https://doi.org/10.1186/s13017-019-0267-9

22. Darrivere L., Lapidus N., Colignon N. et al. (2018). Minimally invasive drainage in critically ill patients with severe necrotizing pancreatitis is associated with better outcomes: an observational study. Critical Care 2018. 22: 321. P. 299-321.

23. Sion M.K., Davis K.A. (2019). Step-up approach for the management of pancreatic necrosis: a review of the literature. Trauma Surgery \& Acute Care Open. 4:e000308. 5 p. DOI:10.1136/tsaco-2019000308

24. Yip H.C, Teoh A.Y.B. (2017). Endoscopic management of peripancreatic fluid collections. Gut and Liver, Published online. Sep 15; 11(5):604-611. https://doi.org/10.5009/gnl16178. May 12.

25. Haas B, Nathens A.B. (2010). Surgical indications in acute pancreatitis. Current Opinion in Critical Care. 16(2). P. 153-158.

26. Hollemans A et al. (2016). Predicting success of catheter drainage in infected necrotizing pancreatitis. Ann Surg. 263(4). P. 787-792. doi: 10.1097/SLA.0000000000001203.

27. Lichi K et al. (2016). Percutaneous Catheter Drainage in Infected Pancreatitis Necrosis: a Systematic Review. Indian J Surg. 78(3). P. 221-228. doi: 10.1007/s12262-016-1495-9

28. Mittu J. M. (2014). Laparoscopic necrosectomy in acute necrotizing pancreatitis: Our experience. J Minim Access Surg. 10(3). P. 126-131. doi: 10.4103/0972-9941.134875.

29. Dua M. M et al. (2015). Surgical Strategies for the Management of Necrotizing Pancreatitis. J Pancreas. 16(6). P. 547-558. doi: 10.3748/wjg.v7.i4.476

30. Pedro S-V, Ana MA, Miguel C-B, Ant?nio G, Maria FB, Jos? GT. (2019). Murine Models of Acute Pancreatitis: A Critical Appraisal of Clinical Relevance. Int J Mol Sci. Jun; 20(11): 2794. Published online 2019 Jun 7. doi: 10.3390/ijms20112794 PMCID:PMC6600324 
31. Porcu M, Solinas C, Migali C, Battaglia A et al. (2020). Immune Checkpoint Inhibitor-Induced Pancreatic Injury: Imaging Findings and Literature Review. Target Oncol. 2020 Feb; 15(1):25-35. doi: 10.1007/s11523-019-00694-w.PMID: 31925647 Review.

32. Rompianesi G, Hann A, Komolafe O, Pereira SP, Davidson BR, Gurusamy KS. (2017). Serum amylase and lipase and urinary trypsinogen and amylase for diagnosis of acute pancreatitis. Cochrane Database Syst Rev. 2017 Apr 21;4(4):CD012010. doi: 10.1002/14651858.CD012010.pub2.PMID: 28431198 Free PMC article. Review.

33. Sorrentino L, Chiara O, Mutignani M, et al. (2017). Combined totally mini-invasive approach in necrotizing pancreatitis: a case report and systematic literature review. World J Emerg Surg. 2017 Mar 16;12:16. doi: 10.1186/s13017-017-0126-5. eCollection 2017.PMID: 28331537

34. Tawfik K, Anas K, Mahmud M, Amir M, Wisam S. (2020). Bedside score predicting retained common bile duct stone in acute biliary pancreatitis. World J Clin Cases. 2020 Apr 26; 8(8): 1414-1423. Published online 2020 Apr 26. doi: 10.12998/wjcc.v8.i8.1414. PMCID:PMC7190963

35. Tonolini M, Di Pietro S. (2019). Diffusion-weighted MRI: new paradigm for the diagnosis of interstitial oedematous pancreatitis. Gland Surg. 2019 Apr;8(2):197-206. doi: 10.21037/gs.2018.12.08. PMID: 31183329 Free PMC article.

36. Weihao K, Yuanyuan H, Huarui B, Wenlong Z, Xingyu W. (2020). Diagnostic Value of NeutrophilLymphocyte Ratio for Predicting the Severity of Acute Pancreatitis: A Meta-Analysis. Dis Markers. 9731854. Published online 2020 Apr 27. doi: 10.1155/2020/9731854 PMCID: PMC7232731

37. Xu B, Bai B, Sha S, Yu P, et al. (2014). Interleukin-1 $\beta$ induces autophagy by affecting calcium homeostasis and trypsinogen activation in pancreatic acinar cells. Int J Clin Exp Pathol. 7:3620-3631.

38. Xuehua P, Baohai L, Xiaodan S, Shuangdi L et al. (2020). Picroside II Improves Severe Acute Pancreatitis-Induced Intestinal Barrier Injury by Inactivating Oxidative and Inflammatory TLR4-Dependent PI3K/AKT/NF-кB Signaling and Improving Gut Microbiota. Oxid Med Cell Longev. 2020: 3589497. Published online 2020 Apr12. doi: 10.1155/2020/3589497 PMCID: PMC7174951

39. Xu X, Ai F, Huang M. (2020). Deceased serum bilirubin and albumin levels in the assessment of severity and mortality in patients with acute pancreatitis. Int J Med Sci. 2020 Sep 23;17(17):2685-2695. doi: 10.7150/ijms.49606. eCollection 2020.PMID: 33162796 Free PMC article.

40. Yasmine HH, Ahmed OH, Abd-Elrahman MM, Joseph RA, Yasser AO. (2020). Adherence to the evidence-based guidelines in the management of acute biliary pancreatitis: A case series. Int J Surg Case Rep. 77: 906-914. Published online 2020 Dec. 2. doi: 10.1016/j.ijscr.2020.11.135 Correction in: Int J Surg Case Rep. 2021 Jan; 78: 417. PMCID:PMC7749290

41. Zhi Z, Yi-Xuan D, Yuan-Xu Q, Feng C, Fei L. (2021). A narrative review of acute pancreatitis and its diagnosis, pathogenetic mechanism, and management. Ann Transl Med. 2021 Jan; 9(1): 69. doi: 10.21037/atm-20-4802 PMCID:PMC7859757

42. Osuka A, Kusuki H, Matsuura H, Shimizu K et al. (2017). Acute intestinal damage following severe burn correlates with the development of multiple organ dysfunction syndrome: A prospective cohort study. 2017 Jun; 43(4):824-829. doi: 10.1016/j.burns.2016.10.015. Epub 2016 Dec 28.PMID: 28040364

43. Michal Z, Georg B, Julia M. (2019). Risk Stratification and Early Conservative Treatment of Acute Pancreatitis. Visc Med. 2019 Apr; 35(2): 82-89. Published online 2019 Mar.25. doi: 10.1159/ 000497290 PMCID:PMC6514505

44. Lingling J, Hao C, Jun Y, Xin F et al. (2020). Combinatory antibiotic treatment protects against experimental acute pancreatitis by suppressing gut bacterial translocation to pancreas and inhibiting NLRP3 inflammasome pathway. Innate Immun. 2020 Jan; 26(1): 48-61. Published online 2019 Oct 15. doi: 10.1177/1753425919881502 PMCID: PMC6974879

45. Heckler M., Hackert Th., Hu K. et al. (2020). Severe acute pancreatitis: surgical indications and treatment (review). Langenbecks Arch. Surg. Sep.10. 2020. doi: 10.1007/s00423-020-01944-6. PMID: 32910276.

46. Amas Gomez L, Zubia Olaskoaga F. (2019). Results of the modification of an acute pancreatitis management protocol in Intensive Care medicine. Med Intensiva. 2019 Dec;43(9):546-555.

47. Adarsh PS, Moustafa MM, Simon RB. (2018). Acute pancreatitis: current perspectives on diagnosis and management. J Inflamm Res. 11: 77-85. Published online 2018 Mar 9. doi: 10.2147/JIR.S135751 PMCID: PMC5849938 
48. Fonseca Sepulveda EV, Guerrero-Lozano R. (2019). Acute pancreatitis and recurrent acute pancreatitis: an exploration of clinical and etiologic factors and outcomes. J Pediatr (Rio J). 2019 Nov Dec; 95(6):713-719.

49. Linlin C, Huidan D, Hengmin C, Jing F. Et al. (2018). Inflammatory responses and inflammationassociated diseases in organs. Oncotarget. 2018 Jan 23; 9(6): 7204-7218. Published online 2017 Dec 14. doi: 10.18632/oncotarget.23208 PMCID:PMC5805548

50.Kehlet H., Wilmore D.W. (2005). Fast-track surgery. Section of Surgical Pathophysiology, The Juliane Marie Centre 4074, Rigshospitalet, DK-2100 Copenhagen, Denmark. P. 3-4. DOI:10.1002/bjs.4841

Received: 23-Feb-2021

Accepted: 11-Jun-2021 